\title{
Inflação Inercial como um Processo de Longa Memória: Análise a partir de um Modelo Arfima-Figarch ${ }^{\star}$
}

- ERIK Alencar de Figueiredo*

\author{
- ANDRÉ M. MARQUES **
}

\begin{abstract}
RESUMO
O objetivo principal deste estudo é investigar a dependência de longo prazo da inflação brasileira, descrevendo-a como um processo fracionariamente integrado tanto na média quanto na variância. A metodologia empregada baseia-se na estimação de um modelo ARFIMA-FIGARCH, capaz de detectar a presença de memória longa em altas defasagens de um processo autorregressivo. Os principais resultados alcançados indicam que, para o período pós-Plano Real, a inflação brasileira exibe um comportamento estacionário em seus dois primeiros momentos com lento decaimento hiperbólico. Há indícios de longa memória na média e na variância do processo. Além disso, constatou-se, para esse período, uma recíproca influência entre a volatilidade e a taxa média de inflação.
\end{abstract}

Palavras-Chave

inflação inercial, ARFIMA-FIGARCH, memória longa, volatilidade

\section{ABSTRACT}

The aim of this paper is search for the long memory in the Brazilian inflation rate, describing it as a fractionally integrated process in the first and second moments. So, it is employed the more recent methodology of ARFIMA-FIGARCH models. The main result endorses the hypothesis of inertial inflation in the short and long run, and the Friedman's hypothesis of interaction between mean and volatility of price inflation.

KEYWORDS

inertial inflation, ARFIMA-FIGARCH, long memory, volatility

\section{JEL Classification}

C22, E3I

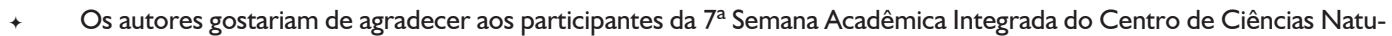
rais e Exatas - CCNE da Universidade Federal de Santa Maria, realizada em agosto de 2007. Em especial, aos professores Paulo S. Ceretta e Adriano M. Souza. Estamos em débito com dois pareceristas anônimos. Erros e omissões que persistam são de responsabilidade dos autores.

* Departamento de Economia da UFPB. Endereço para contato: Centro de Ciências Sociais Aplicadas, Universidade Federal da Paraíba - Cidade Universitária - Campus I - João Pessoa - PB. CEP: 58059-900. E-mail: eafigueiredo@ gmail.com.

** Departamento de Economia da UFRN. Centro de Ciências Sociais Aplicadas - UFRN - Campus Universitário, Av. Senador Salgado Filho, s/n - Natal - RN. CEP: 59072-970. E-mail: andremmarques@yahoo.com.br.

(Recebido em agosto de 2007. Aceito para publicação em julho de 2008). 


\section{$1 \quad$ INTRODUÇÃO}

Quaisquer que sejam as suas causas efetivas, as alterações nos preços relativos quase sempre expressam desequilíbrios reais e provocam alterações no comportamento dos agentes econômicos. Nesse particular, a variação do nível geral de preços em uma economia e o caráter de sua resposta a impulsos inflacionários ou deflacionários (choques) são um aspecto de grande importância tanto para a teoria quanto para a política macroeconômica.

A inflação brasileira, no contexto atual, está estabilizada em torno de $4 \%$ ao ano. Abstraindo sua história pregressa, o Brasil tem hoje uma taxa de inflação próxima à dos países avançados. Contudo, seria importante ter presente nas considerações atuais de política uma medida mais precisa da longa memória (inércia) da inflação brasileira, bem como o real caráter de sua resposta a eventuais choques aleatórios.

Convencionalmente, para explicar a variação do nível geral de preços, a teoria econômica adota a suposição de excesso de moeda na economia. Contudo, no caso brasileiro, a explicação teórica ${ }^{1}$ e os trabalhos empíricos realizados tendem a corroborar o caráter marcadamente inercial da taxa de inflação. A hipótese da inflação inercial foi formulada sobre bases (teóricas e empíricas) genuinamente domésticas. A experiência brasileira demonstrou o realismo dessa suposição e o acerto de suas premissas com o sucesso alcançado pelo Plano Real. ${ }^{2}$

Para compreendê-la, faz-se normalmente a clara distinção entre choque e tendência inflacionária. Suponha que seja possível medir exatamente a contribuição de todos os choques (oferta agregada, overshooting cambial, abertura econômica, demanda agregada etc) sobre a taxa de inflação observada. A componente isolada não explicada pelos choques será então a tendência inflacionária. Na ausência de choques, a taxa de inflação corrente será igual a essa tendência.

A explicação dominante encontrada nos livros-texto para a tendência inflacionária, assim definida, apoia-se em um forte componente expectacional: a expectativa de inflação formada pelos agentes econômicos condiciona a taxa de inflação corrente e, por extensão, a taxa de inflação futura; por isso, o Banco Central deposita grande peso sobre a expectativa de inflação formada pelos agentes econômicos (BOGDANSKI et. al., 2000). Entretanto, a hipótese da inflação inercial adota um suposto diferente para explicar a tendência inflacionária: toda alteração na tendência inflacionária resulta de ações de agentes econômicos visando recompor seu pico de renda real obtido no passado. Isto é, na ausência de choques ou impulsos infla-

1 Em especial, Modiano (1983), Lopes (1985) e Resende (1985).

2 Sobre a elaboração e implementação do Plano Real, ver Bacha (1998). 
cionários (deflacionários), a inflação corrente é determinada pela inflação passada, independentemente das expectativas (LOPES, 1985; RESENDE, 1985).

Quando esse comportamento defensivo é suficientemente ampliado na economia, a inflação observada na ausência de choques é idêntica à inflação passada. Em ambientes cronicamente inflacionados, esse comportamento defensivo dos agentes econômicos tende a se perpetuar por longos períodos com ou sem os mecanismos institucionais de indexação.

Neste contexto, a asserção de Friedman (1976) relacionando a taxa de inflação e a volatilidade adquire certa importância. Embora essa associação refira-se a ambientes de hiperinflação, ela poderá ser contextualizada em um ambiente de inflação inercial uma vez que toda inflação crônica é predominantemente inercial (LOPES, 1985). Em essência, Friedman observou que, em economias de alta inflação, o ambiente de incerteza tende a provocar um certo comportamento nos agentes econômicos de modo que quanto maior a taxa de inflação, maior a sua volatilidade, podendo persistir por longos períodos: "(...) a high inflation rate is not likely to be steady during the transition decades. Rather, the higher the rate, the more variable it is likely to be" (FRIEDMAN, 1976).

Muitos estudos sobre o processo inflacionário no Brasil já foram realizados desde o começo da década de 1980, tendo como foco importante o mercado de trabalho e a política salarial. No geral, os achados empíricos ofereciam suporte a uma Curva de Phillips marcadamente horizontal, inviabilizando qualquer tentativa de conter a inflação pelos instrumentos convencionais de restrição da demanda agregada (LOPES, 1985; RESENDE, 1985).

Já nos anos 1990, os estudos empíricos vêm endossando a interpretação de que a inflação brasileira, mesmo após a implantação do Plano Real, continua com uma importante componente inercial, apesar de muitos dos mecanismos institucionais de indexação terem sido removidos nos últimos anos. Não é estranho, por isso, que a taxa de inflação apresente um caráter persistente não somente em curtos períodos, mas também em longos períodos.

Os resultados do estudo de Figueiredo e Ferreira (2002), por exemplo, indicam que a depreciação cambial influencia com maior intensidade os preços indexados, administrados pelo governo, que os preços livres. Segundo os autores, mais de 51\% da inflação (IPCA) do período 1999-2002 é explicada exclusivamente pelos preços indexados. O estudo de Fasolo e Portugal (2003), estimando uma Curva de Phillips não-linear para a economia brasileira, constata a alta persistência (inércia) da inflação 
brasileira para o período 1990-2002, concluindo que ela possui uma componente inercial autônoma, independente de quaisquer choques aleatórios.

Cati et al. (1999), empregando técnicas de raiz unitária com controle para inliers, constataram o caráter predominantemente não estacionário da inflação brasileira para o período 1974-1993, isto é, a possibilidade de incorporação permanente de influências exógenas sobre sua trajetória de longo prazo. Impulsos inflacionários (ou deflacionários) tendem a afetar de forma permanente a trajetória de longo prazo do processo inflacionário. Estes resultados foram posteriormente corroborados, em maior ou menor grau, por Tejada e Portugal (2001), Campêlo e Cribari-Neto (2003) e Cribari-Neto e Cassiano (2005).

Recentemente, Laurini e Vieira (2005) destacaram que a dinâmica inflacionária brasileira, no pós-Plano Real, apresenta um componente de heterocedasticidade condicional. Por conta disso, os autores propõem um modelo de variação dos parâmetros ao longo do tempo, com erros GARCH (Generalized Autoregressive Conditional Heteroskedasticity). Os resultados do estudo apontam para um crescimento da inércia em períodos de crises, associando-a ao comportamento da volatilidade inflacionária. Porém, afora a relevância dos resultados, destaca-se a pertinência de uma caracterização não-linear para a série.

Tendo esses resultados como suporte, o objetivo principal deste artigo é investigar a memória longa da inflação brasileira, descrevendo-a como um processo fracionariamente integrado tanto na média quanto na variância. Para isso, estima-se um modelo ARFIMA-FIGARCH ${ }^{3}$ capaz de detectar tanto a memória de curto prazo quanto a memória de longo prazo nos dois primeiros momentos de uma variável. Ademais, este modelo capta o comportamento não-linear da série. Os dados mensais vão de 1980 a 2008, com estimativas para alguns subperíodos históricos. Adicionalmente, o estudo também verifica se há alguma relação definida entre a média e a volatilidade da inflação, tal como foi sugerido por Friedman (1976).

Os principais resultados alcançados no trabalho indicam que, para o período pósPlano Real, a inflação brasileira exibe um comportamento estacionário em seus dois primeiros momentos com lento decaimento hiperbólico. Há indícios de longa memória na média e na variância do processo. Além disso, constatou-se, para esse período, uma recíproca influência entre a volatilidade e a taxa média de inflação.

3 Ver Sowell (1992) para uma discussão teórica. Os modelos ARIMA podem ser considerados modelos de "memória curta", enquanto os modelos ARFIMA captam além da memória de curto prazo, a memória de longo prazo, representada pelo coeficiente $d, 0<d<1$. Para uma síntese dessa literatura, ver Baillie et. al. (1996a). 
Por fim, o artigo está organizado do seguinte modo: na segunda seção, é apresentado o modelo teórico a ser estimado; na terceira seção, têm-se os resultados das estimativas e, na seção quatro, são desenvolvidos os comentários finais.

\section{MODELO ARFIMA-FIGARCH}

De acordo com Granger e Joyeux (1980), um modelo fracionário na média pode ser representado por:

$$
a(L)(1-L)^{d_{m}} y_{t}=\mu+b(L) u_{t}
$$

Com $u_{t} \sim \operatorname{iid}\left(0, \sigma^{2}\right)$. Em que $a(L)=1-a_{1} L-\ldots-a_{p} L^{p}$ e $b(L)=1-b_{1} L-\ldots-b_{q} L^{q}$ correspondem, respectivamente, aos componentes autorregressivos e de médias móveis, todos fora do círculo unitário. O parâmetro $d_{m}$ captura a longa memória do processo. Desde que $\operatorname{VAR}\left(u_{t}\right)<\infty$ e $-0,5<d_{m}<0,5$, o processo $\left\{y_{t}\right\}$ na equação (2.1) será fracamente estacionário e inversível.

Considere agora que o modelo (2.1) apresente resíduos $\operatorname{GARCH}(r, s)^{4}$ e não mais iid $\left(0, \sigma^{2}\right)$, e também que a equação da variância condicional de $\left\{u_{t}\right\}$ exiba, da mesma forma que a média, um comportamento fracionário. Assim, o complemento de (2.1) será:

$$
\begin{aligned}
& u_{t} \sim \sqrt{h_{t}} \varepsilon_{t} \\
& \varphi(L)(1-L)^{d_{v}} h_{t}=\omega+[1-\beta(L)] \varepsilon_{t}^{2}
\end{aligned}
$$

Neste caso, $\varepsilon_{t} \sim \operatorname{iid}\left(0, \sigma^{2}\right)$, em que $0<d_{v}<1$ e todas as raízes das polinomiais $\varphi(L)$ e $[1-\beta(L)]$ estão situadas fora do círculo unitário. O processo descrito pela equação (2.3) será ergódico e estacionário para $0 \leq d_{v} \leq 1$. O sistema geral (2.1), (2.2) e (2.3) caracteriza o modelo ARFIMA-FIGARCH.

Baillie et al. (2002) e Baillie et al. (1996a) discutem uma importante característica deste modelo: sua variância incondicional será infinita desde que $d_{v} \neq 0, \forall d_{m}$. Ou

4 Para detalhes sobre os modelos GARCH, ver Bollerslev (1986). 
seja, a dinâmica desta variância será independente do comportamento da média condicional.

Assumindo-se a normalidade, o logaritmo da verossimilhança para o processo (2.1) e (2.3) será:

$$
L\left(\lambda, u_{1}, \ldots, u_{T}\right)=-(T / 2) \ln (2 \pi)-(1 / 2) \sum_{t=1}^{T}\left[\ln \left(h_{t}^{2}\right)+u_{t}^{2} h_{t}^{-2}\right] .
$$

Onde $\lambda^{\prime}=\left(\mu, \phi_{1}, \ldots, \phi_{p}, \theta_{1}, \ldots, \theta_{q}, d_{m}, w, \beta, v, d_{v}\right)$. As condições para a maximização são descritas em Baillie et al. (1996a). Em geral, o processo de maximização de (2.4) envolve problemas de otimização não-linear, pois o estimador não possui forma fechada. Sendo assim, (2.4) precisa ser otimizada numericamente. Em resumo, o modelo ARFIMA-FIGARCH busca capturar uma dupla longa memória, ou seja, na média e na variância condicionais. Em face de sua estrutura complexa, é interessante selecionar uma baixa defasagem em seu processo de estimação.

\section{RESULTADOS}

A apresentação dos resultados seguirá a seguinte estratégia: a) em primeiro lugar, discutem-se os dados e a consideração de subamostras; b) em seguida, proceder-se-á a uma análise preliminar, baseada nas funções de autocorrelação e nos testes para raiz unitária. Com isso, espera-se detectar indícios de não-estacionariedade e sobrediferenciação da série; c) o terceiro passo consiste em estimar o coeficiente fracionário, com uma posterior análise dos resíduos das regressões. Neste tópico, buscam-se evidências relacionadas à presença de heterocedasticidade condicional e; d) por fim, apresentam-se as estimativas para o modelo ARFIMA-FIGARCH.

\subsection{Dados}

A inflação brasileira será representada pelo Índice Geral de Preços - Disponibilidade Interna (IGP-DI), calculado pela Fundação Getúlio Vargas. Este índice representa uma média ponderada de três outros indicadores: 60\% do Índice de Preços no Atacado (IPA); 30\% do Índice de Preços ao Consumidor (IPC), mensurado a partir da variação de preços das cestas de consumo das famílias que recebem de 1 a 33 salários mínimos e; 10\% do Índice Nacional da Construção Civil (INCC). Os dados foram obtidos junto ao endereço eletrônico do Instituto de Pesquisa Econômica 
Aplicada - IPEA. ${ }^{5}$ Este tem sido o índice de preços utilizado para os trabalhos dessa natureza no Brasil, em especial por Campêlo e Cribari Neto (2003), Cribari Neto e Cassiano (2005) e Maia e Cribari Neto (2006). A utilização do índice de preços ao consumidor amplo (IPCA) em trabalho futuro, pode dar importantes indicações sobre o regime de metas de inflação; porém, neste contexto, onde se busca evidência sobre o comportamento mais agregado dos agentes econômicos, a sua utilização imporia um caráter mais restritivo ao trabalho, visto que sua variação expressa somente a inflação medida nos bens de consumo de um certo número de famílias, omitindo a influência dos preços do atacado e da construção civil.

Foram consideradas as observaçóes mensais compreendidas entre 1980:01 e 2008:01. A evolução da série pode ser observada no Gráfico 1.

\section{GRÁFICO I - EVOLUÇÃO DA INFLAÇÃO BRASILEIRA: 1980:01 A 2008:01}

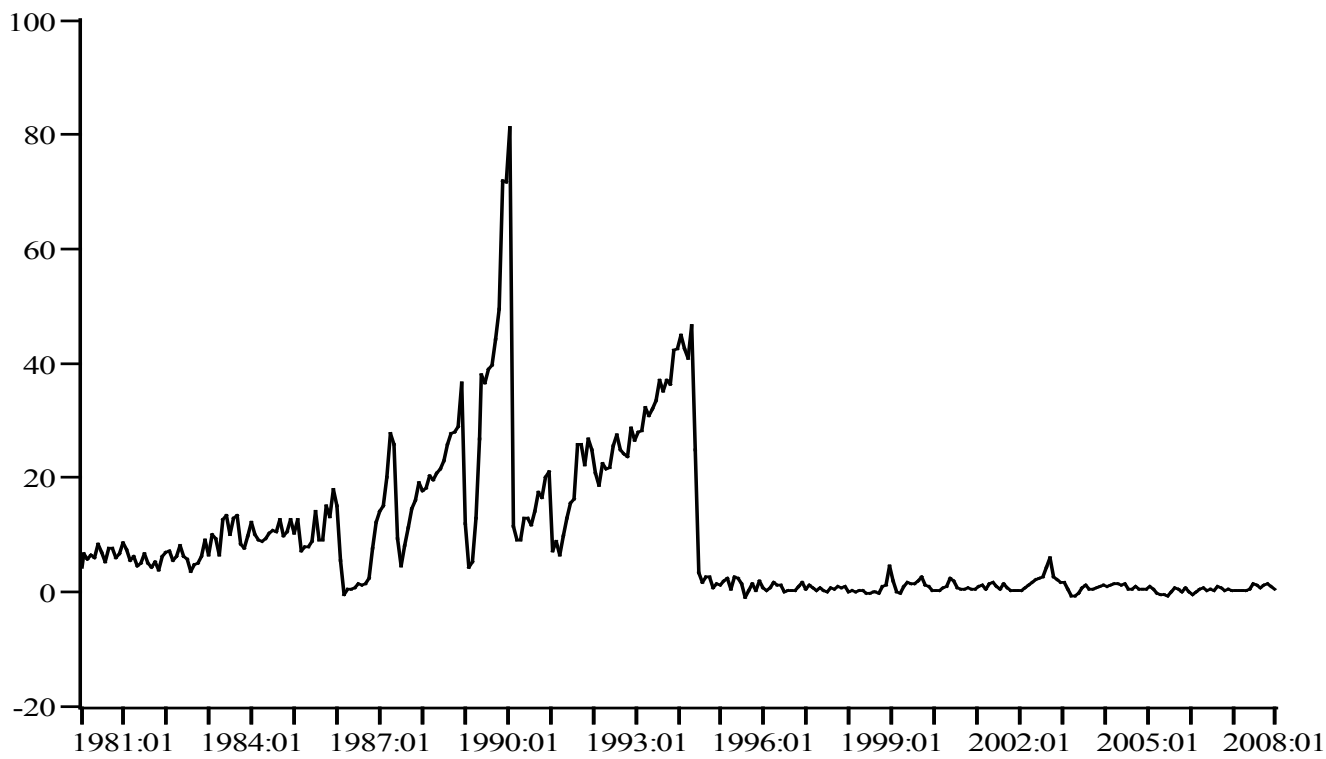

Fonte: Dados da Pesquisa.

A inspeção visual indica a presença de, no mínimo, dois regimes. O primeiro, caracterizado pela contínua elevação do índice inflacionário e o segundo, caracterizado pela estabilização dos preços. Por conta desta heterogeneidade, optou-se por considerar quatro subamostras: a primeira, Al, inclui todo o período: 1980:01-2008:01 (com possíveis quebras e inliers), compreende 337 observações; A2: 1980:01-1991:06, contendo inliers, seguindo o trabalho de Cati et. al. (1999), possui 138 observaçóes; A3: 1991:10-2008:01, sem inliers e com uma provável mudança no intercepto e na

5 http://www.ipeadata.gov.br. 
tendência, compreendendo 196 observações; e, finalmente A4: 1994:08-2008:01, período pós-Plano Real, sem quebras e sem inliers, com 162 observações.

Convém destacar que as subamostras Al e A2 possuem fortes influências de inliers, ou seja, planos de estabilização que atuam de forma transitória sobre a trajetória de longo prazo da série. De acordo com Cati et. al. (1999) e Campêlo e Cribari-Neto (2003), esta característica pode viesar algumas estatísticas tradicionais, notadamente os testes para raiz unitária e as medidas de persistência. Por isso, os testes de raiz unitária para os subperíodos Al e A2 devem incorporar um controle para inliers, a exemplo do trabalho de Cati et. al. (1999). Em caso contrário, os testes de raiz unitária convencionais (ADF, PP) são severamente viesados em favor da rejeição da hipótese de raiz unitária quando de fato a série exibe comportamento não estacionário ou explosivo (CATI et. al., 1999).

\subsection{Análise Preliminar}

Em geral, a observação dos coeficientes de autocorrelação para série da inflação brasileira (Tabela 1) não apresenta evidências explícitas relacionadas à presença ou não de estacionariedade. Em resumo, ela sugere: a) a não-estacionariedade na primeira subamostra; b) a estacionariedade da série no período $\mathrm{A} 4$, devido à queda rápida das autocorrelações e; c) um comportamento inconclusivo para os demais subperíodos.

Este comportamento já era esperado, pois a determinação da ordem de integração da série da inflação brasileira envolve uma vasta discussão em torno da robustez dos testes estatísticos, não sendo possível determiná-la a partir de uma simples análise de autocorrelação. Não obstante a simplicidade deste procedimento, a observação destas estatísticas pode trazer importantes informações sobre a série estudada.

A Tabela 2 apresenta os coeficientes de autocorrelação para a primeira diferença da inflação. Note que, em A2 e A4, a primeira autocorrelação apresenta um valor negativo, indicando uma possível sobrediferenciação da série. 


\section{TABELA I - AUTOCORRELAÇÕES PARA A SÉRIE DE INFLAÇÃO BRASILEIRA}

\begin{tabular}{lcccc}
\hline \multirow{2}{*}{ Defasagens } & \multicolumn{5}{c}{ Inflação - IGP - DI } \\
\cline { 2 - 5 } & $\mathrm{A} 1$ & $\mathrm{~A} 2$ & $\mathrm{~A} 3$ & $\mathrm{~A} 4$ \\
\hline 1 & 0,9122 & 0,8243 & 0,9666 & 0,6338 \\
2 & 0,8194 & 0,6511 & 0,9242 & 0,3638 \\
3 & 0,7279 & 0,4764 & 0,8819 & 0,2292 \\
4 & 0,6733 & 0,3930 & 0,8403 & 0,1780 \\
5 & 0,6306 & 0,3445 & 0,7958 & 0,0907 \\
6 & 0,5974 & 0,3130 & 0,7550 & $-0,0061$ \\
7 & 0,5626 & 0,2692 & 0,7174 & $-0,0364$ \\
8 & 0,5331 & 0,2293 & 0,6847 & $-0,0279$ \\
9 & 0,4982 & 0,1764 & 0,6514 & 0,0241 \\
10 & 0,4797 & 0,1668 & 0,6145 & $-0,0215$ \\
11 & 0,4816 & 0,2026 & 0,5769 & $-0,0424$ \\
12 & 0,4865 & 0,2411 & 0,5486 & $-0,0563$ \\
\hline
\end{tabular}

Fonte: Dados da pesquisa.

TABELA 2 - AUTOCORRELAÇÕES PARA A PRIMEIRA DIFERENÇA DA SÉRIE DE INFLAÇÃO BR ASILEIRA

\begin{tabular}{lcccc}
\hline \multirow{2}{*}{ Defasagens } & \multicolumn{5}{c}{ Primeira Diferença da Inflação - IGP - DI } \\
\cline { 2 - 5 } & $\mathrm{A} 1$ & $\mathrm{~A} 2$ & $\mathrm{~A} 3$ & $\mathrm{~A} 4$ \\
\hline 1 & 0,0311 & $-0,0044$ & 0,2376 & $-0,1369$ \\
2 & $-0,0073$ & 0,0031 & $-0,0753$ & $-0,1834$ \\
3 & $-0,2131$ & $-0,2618$ & 0,0142 & $-0,1032$ \\
4 & $-0,0687$ & $-0,1012$ & 0,1169 & 0,0427 \\
5 & $-0,0535$ & $-0,0448$ & $-0,0389$ & 0,0166 \\
6 & 0,0089 & 0,0334 & $-0,1282$ & $-0,0955$ \\
7 & $-0,0321$ & $-0,0138$ & $-0,0871$ & $-0,0612$ \\
8 & 0,0318 & 0,0406 & 0,0050 & $-0,0470$ \\
9 & $-0,0946$ & $-0,1253$ & 0,0094 & 0,1182 \\
10 & $-0,1178$ & $-0,1330$ & $-0,0174$ & $-0,0317$ \\
11 & $-0,0173$ & $-0,0067$ & $-0,1494$ & $-0,0026$ \\
12 & 0,0204 & 0,0080 & 0,0415 & $-0,0337$ \\
\hline
\end{tabular}

Fonte: Dados da pesquisa. 
Para tornar os resultados mais robustos, optou-se pela implementação dos testes para raiz unitária. Entretanto, deve-se destacar que, de acordo com Schwert (1989), os testes tradicionais sofrem importantes distorções quando a primeira diferença da série apresenta um componente de médias móveis negativo e próximo a um. Por conta disso, o primeiro passo será estimar um ARIMA $(0,1,1)$. Esta característica, aliada à presença de outliers e inliers, pode dificultar a identificação do processo gerador dos dados. Por essa razão, adotou-se uma estratégia de estimação envolvendo três procedimentos: os testes Phillips e Perron (1988) - PP, Kwiatkowski et al. (1992) KPSS e Zivot e Andrews (1992) - ZA.

$\mathrm{O}$ teste de PP baseia-se em uma abordagem semiparamétrica a partir do princípio da invariância e dos funcionais movimentos brownianos, considerando que os termos de perturbação estocástica são serialmente correlacionados. Assim como a maioria dos testes clássicos para estacionariedade, o procedimento PP possui como hipótese nula a existência de uma raiz unitária. ${ }^{6}$ Já o teste KPSS possui como hipótese nula a inexistência de raiz unitária.

O teste de ZA possui como hipótese nula a existência de raiz unitária, e, diferente dos demais testes acima, possui como hipótese alternativa a estacionariedade com um componente determinístico "flexível". Isto é, o teste incorpora a possibilidade de alterações no intercepto e/ou na tendência da série, estimando recursivamente o ponto da quebra a partir das próprias observações.

Espera-se que a utilização conjunta dos três testes possibilite uma determinação mais precisa da ordem de integração da série. De acordo com Baillie et al. (1996b), a utilização conjunta dos testes PP e KPSS pode gerar quatro resultados:

a) a rejeição da hipótese nula do teste PP e a não rejeição da hipótese nula do teste KPSS, indicando forte evidência de um processo de covariância-estacionária;

b) a não rejeição da hipótese nula do teste PP e a rejeição da hipótese nula do teste KPSS, indicando que a série possui uma raiz unitária;

c) a não rejeição das hipóteses nulas em ambos os testes, o que gera uma indeterminação sobre o processo gerador dos dados e;

d) a rejeição da hipótese nula em ambos os testes, sugerindo que o processo gerador não é $\mathrm{I}(0)$ nem $\mathrm{I}(\mathrm{l})$, indicando uma provável integração fracionária.

6 Este tem sido um ponto de crítica para a inferência bayesiana, por exemplo. Ou seja, os testes clássicos tendem a tomar a hipótese da raiz unitária como verdadeira. Em contrapartida, os estudos bayesianos vislumbram a simetria com que as hipóteses nula e alternativa são tratadas. Para detalhes, ver Sims (1988). 
Os resultados dos testes PP, KPSS e ZA podem ser observados na Tabela 3. Em um primeiro momento, destacam-se os componentes de médias móveis para o modelo $\operatorname{ARIMA}(0,1,1)$. Note que há valores negativos em A2 e A4, mas que não estão próximos da unidade. Nos testes de raiz unitária foram considerados dois processos geradores: um com constante (..) e o outro com constante e tendência (.. ${ }^{\mathrm{CT}}$. Optouse por estas caracterizações por não se ter claramente um processo gerador bem definido em alguns períodos.

Os resultados indicam que: i) para os subperíodos Al e A2 há uma aparente contradição, pois os testes PP e KPSS apresentam resultados contraditórios e inconclusivos; ii) as subamostras A3 e A4 caracterizam-se pela covariância-estacionária; iii) para A3, especificamente, a aplicação direta dos testes convencionais PP e KPSS resulta na não rejeição da hipótese nula de raiz unitária. Não obstante, quando a mudança no intercepto e na tendência da inflação é explicitamente permitida no modelo, a hipótese de raiz unitária é rejeitada a $1 \%$ de significância. ${ }^{7}$

TABELA 3 - RAIZ UNITÁRIA PARA A INFLAÇÃO BRASILEIRA

\begin{tabular}{|c|c|c|c|c|c|c|c|}
\hline \multirow{2}{*}{ Período } & \multicolumn{7}{|c|}{ Inflação Brasileira } \\
\hline & MA & $\mathrm{PPC}$ & PPCT & $\mathrm{KPSS}^{\mathrm{C}}$ & $\mathrm{KPSS}^{\mathrm{CT}}$ & $Z A^{C}$ & $\mathrm{ZA}^{\mathrm{CT}}$ \\
\hline \multirow[t]{2}{*}{$\mathrm{A} 1$} & 0,0310 & $-3,6674^{* \star *}$ & $-4,0777^{\star \star \star}$ & $0,6638^{* \star}$ & $0,2502^{* * *}$ & -..-- & -..-- \\
\hline & $(0,0538)$ & & & & & ----- & ------ \\
\hline \multirow[t]{2}{*}{ A2 } & $-0,0044$ & $-3,4486^{\star \star}$ & $-3,8232^{\star \star}$ & $0,5952^{* *}$ & 0,0791 & ----- & -.---. \\
\hline & $(0,0852)$ & & & & & ----- & -.--- \\
\hline \multirow[t]{2}{*}{ A3 } & $0,3035^{\star \star *}$ & $-2,1637$ & $-2,2992$ & $0,6875^{\star \star}$ & $0,2072^{* *}$ & $-11,6112^{\star * *}$ & $-22,3202^{* * *}$ \\
\hline & $(0,0718)$ & & & & & $(1994: 05)$ & $(1994: 05)$ \\
\hline \multirow[t]{2}{*}{ A4 } & $-0,2616^{\star \star \star}$ & $-5,7496^{\star \star \star}$ & $-5,7875^{\star \star \star}$ & 0,1718 & 0,0891 & ----- & ----- \\
\hline & $(0,1100)$ & & & & & & \\
\hline
\end{tabular}

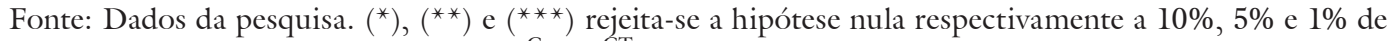
significância. Os sobrescritos $(.)^{\mathrm{C}} \mathrm{e}(.)^{\mathrm{CT}}$ denotam as especificaçốes "com constante" e "com constante e tendência”, respectivamente. Erros padronizados entre parênteses.

Como já ressaltado, a análise para as séries nacionais de taxa de inflação da década de 1980 apresenta uma dificuldade adicional devido à existência de inliers, incluindo o período do último plano de estabilização (Collor II: 1991:02-1991:06), conforme documentado no trabalho de Cati et. al. (1999, p. 29), com duração de cinco meses.

7 Ver Montañés et. al. (2005) para uma discussão sobre as consequências da omissão de mudanças estruturais nos testes de raiz unitária. Os coeficientes das variáveis dummies para o intercepto e para a tendência nos modelos estimados (ZIVOT; ANDREWS, 1992, p. 254) são estatisticamente significativos a 1\% de significância, indicando que, como se esperava, houve uma alteração significativa tanto no intercepto quanto na tendência da inflação em 1994:05. As saídas do software utilizado estão disponíveis com os autores, sob demanda. 
Essa característica presente nas subamostras Al e A2 introduz um importante viés no processo de estimação convencional, uma vez que os testes convencionais (PP, ADF) tendem a rejeitar a hipótese nula, sugerindo a estacionariedade e a influência transitória de choques sobre a taxa de inflação. Por isso, os resultados dos testes de raiz unitária PP e KPSS apresentados na Tabela 3 não podem ser conclusivos nem dar uma indicação precisa da ordem de integração da série de inflação para as subamostras A1 e A2, posto que não incluem um controle para inliers.

Outro viés é introduzido quando a hipótese de raiz unitária é testada desprezandose as possíveis mudanças estruturais no intercepto e/ou na tendência da série. Neste caso, a omissão da possibilidade de quebra introduz um viés no teste de raiz unitária de modo que a hipótese de não-estacionariedade tende a não ser rejeitada, quando na verdade a série é de natureza estacionária com uma provável mudança na tendência e/ou na inclinação (ZIVOT; ANDREWS, 1992 e MONTAÑÉS et. al., 2005). Por isso, sem a presença de inliers, no caso da subamostra A3, essa característica do processo gerador dos dados deve ser explicitamente considerada. A data estimada da quebra (entre parênteses) e a rejeição da hipótese de raiz unitária são resultados obtidos após a aplicação do teste de Zivot e Andrews (1992), tendo em conta o Plano Real em 1994. Note que as grandes dificuldades em determinar o processo gerador dos dados foram encontradas justamente nos períodos onde se observam os inliers (A1 e A2). ${ }^{8}$

\subsection{Modelos ARFIMA}

Os resultados da seção anterior não foram muito conclusivos, porém, destaca-se o comportamento de duas subamostras. Para A2, os resultados dos testes KPSS e PP são contraditórios e não conclusivos; no entanto, na Tabela 2, para essa subamostra há indícios de sobrediferenciação e integração fracionária. Já em A4, os resultados dos testes KPSS e PP indicam que a série é covariância-estacionária, não obstante a Tabela 2 ofereça indicações de sobrediferenciação e integração fracionária nesse subperíodo.

É importante observar que os testes PP e KPSS só exploram os casos limite I(l) ou $\mathrm{I}(0)$, e por isso, podem não captar a presença de integração fracionária de um coeficiente $d$ próximo, porém, estatisticamente diferente de zero. Essa limitação dos testes PP e KPSS já foi constatada por Baillie et. al. (1996b), para o caso da inflação na Alemanha e no Japão, com dados mensais para o período 1948-1990. Por isso,

8 Nesse sentido, Campêlo e Cribari-Neto (2003) conduzem sua investigação sob o teste alternativo de Hasan-Koenker, permitindo uma melhor performance dada a sua não-sensibilidade à presença de inliers. 
nesse contexto, destaca-se a importância da estimação das autocorrelações para A2 e A4, mostradas na Tabela 2.

Por conta disso, esta seção investigará a pertinência de uma caracterização fracionária para as séries. Como uma etapa preliminar, procedeu-se a filtragem fracionária das séries, utilizando-se o operador $(1-L)^{d}$. Devido aos problemas já discutidos anteriormente, em especial a presença de outliers e inliers, optou-se por considerar apenas a amostra A4. A Tabela 4 apresenta os resultados das funções de autocorrelação, considerando diferenciações fracionárias, com o $d$ variando entre 0,1 e $0,9 .{ }^{9}$

TABELA 4 - AUTOCORRELAÇÕES PARA A SÉRIE A4 FILTR ADA

\begin{tabular}{lccccccccc}
\hline \multirow{2}{*}{ Defasagens/d Inflação - IGP - DI (A4) } \\
\cline { 2 - 9 } & 0,1 & 0,2 & 0,3 & 0,4 & 0,5 & 0,6 & 0,7 & 0,8 & 0,9 \\
\hline 1 & 0,546 & 0,456 & 0,366 & 0,279 & 0,197 & 0,121 & 0,050 & $-0,015$ & $-0,075$ \\
2 & 0,256 & 0,157 & 0,070 & $-0,002$ & $-0,061$ & $-0,106$ & $-0,140$ & $-0,163$ & $-0,177$ \\
3 & 0,134 & 0,055 & $-0,008$ & $-0,054$ & $-0,086$ & $-0,106$ & $-0,117$ & $-0,121$ & $-0,120$ \\
4 & 0,110 & 0,061 & 0,030 & 0,014 & 0,008 & 0,011 & 0,018 & 0,027 & 0,038 \\
5 & 0,032 & $-0,006$ & $-0,027$ & $-0,034$ & $-0,032$ & $-0,025$ & $-0,015$ & $-0,004$ & 0,006 \\
6 & $-0,064$ & $-0,102$ & $-0,122$ & $-0,130$ & $-0,129$ & $-0,123$ & $-0,115$ & $-0,106$ & $-0,098$ \\
7 & $-0,084$ & $-0,111$ & $-0,122$ & $-0,122$ & $-0,115$ & $-0,104$ & $-0,092$ & $-0,080$ & $-0,068$ \\
8 & $-0,064$ & $-0,083$ & $-0,089$ & $-0,087$ & $-0,081$ & $-0,075$ & $-0,069$ & $-0,064$ & $-0,061$ \\
9 & 0,010 & 0,018 & 0,025 & 0,043 & 0,063 & 0,082 & 0,099 & 0,113 & 0,124 \\
10 & $-0,044$ & $-0,052$ & $-0,051$ & $-0,046$ & $-0,040$ & $-0,035$ & $-0,032$ & $-0,031$ & $-0,031$ \\
11 & $-0,063$ & $-0,069$ & $-0,066$ & $-0,059$ & $-0,049$ & $-0,039$ & $-0,029$ & $-0,022$ & $-0,015$ \\
12 & $-0,078$ & $-0,086$ & $-0,087$ & $-0,082$ & $-0,076$ & $-0,069$ & $-0,063$ & $-0,057$ & $-0,053$ \\
\hline
\end{tabular}

Fonte: Dados da pesquisa.

Note que as primeiras autocorrelações tornam-se negativas à medida que o $d$ aproxima-se de 1 , indicando a sobrediferenciação da série. Entretanto, para valores do $d$ inferiores a 0,3 a série tende a apresentar um comportamento estacionário, indicando uma boa aproximação em sua diferenciação.

Um resultado mais robusto é apresentado na Tabela 5. Nela, são sumariadas as estimativas para o modelo fracionário na média. Para tanto, utilizou-se um processo de estimação baseado em Haslett e Raftery (1989). O processo de seleção da parte AR e MA (ordem máxima permitida $(\mathrm{p}=5, \mathrm{q}=5)$ ) foi realizado a partir do critério de Akaike (AIC).

9 Utilizou-se o procedimento de diferenciação fracionária proposto por Reisen (1994). As tabelas relacionadas aos demais períodos encontram-se disponíveis com os autores. 
É importante ressalvar que a subamostra A2 contém inliers e mudanças estruturais, por isso, a estimativa do coeficiente $d$ do modelo ARFIMA para esse subperíodo mostrado na Tabela 5 abaixo pode apresentar viés de estimação. Seu valor poderá dar apenas uma ligeira indicação do coeficiente $d$, apenas com finalidade de controle em relação ao coeficiente $d$ estimado para o subperíodo A4, sem quebras ou inliers.

TABELA 5 - MODELOS ARFIMA PARA A INFLAÇÃO BRASILEIRA

\begin{tabular}{|c|c|c|}
\hline Parâmetros & $\mathrm{A} 2$ & A4 \\
\hline$\hat{d}_{m}$ & $\begin{array}{l}0,3596^{\star * *} \\
(0,0567)\end{array}$ & $\begin{array}{c}0,0870^{* *} \\
(0,0454)\end{array}$ \\
\hline$\hat{a}_{1}$ & $\begin{array}{l}-0,4102^{* * *} \\
(0,0832)\end{array}$ & $\begin{array}{l}0,5576^{\star * *} \\
(0,0649)\end{array}$ \\
\hline$\hat{a}_{2}$ & $\begin{array}{l}-0,3274^{\star * *} \\
(0,0841)\end{array}$ & -- \\
\hline$\hat{a}_{3}$ & $\begin{array}{l}0,4126^{\star * *} \\
(0,0799)\end{array}$ & --- \\
\hline$\hat{b}_{1}$ & $\begin{array}{l}0,9743^{\star \star *} \\
(0,0297)\end{array}$ & --- \\
\hline$\hat{b}_{2}$ & $\begin{array}{l}1,0000^{\star \star *} \\
(0,0390)\end{array}$ & -- \\
\hline $\log \ell$ & $-463,51$ & $-177,46$ \\
\hline AIC & 939,02 & 358,93 \\
\hline$Q(4)$ & $(0,8671)$ & $(0,7687)$ \\
\hline$Q(10)$ & $(0,8503)$ & $(0,8000)^{\star}$ \\
\hline$Q(20)$ & $(0,9814)$ & $(0,9569)$ \\
\hline
\end{tabular}

Fonte: Dados da pesquisa. Erro padrão entre parênteses. $\left({ }^{*}\right),\left({ }^{* *}\right) \mathrm{e}\left({ }^{* * *}\right)$ denotam, respectivamente, $10 \%$, $5 \%$ e $1 \%$ de significância estatística. Probabilidade exata do teste Q (.) Ljung-Box para os resíduos da regressão entre parênteses.

Em primeiro lugar, destaca-se a significância estatística dos coeficientes fracionários. Os valores de $d$ inferiores a 0,5 sugerem a estacionariedade das séries. Porém, embora os erros sejam não-correlacionados em todos os modelos (a 1\% e 5\% de significância), como indicam os resultados do teste de Ljung-Box, os quadrados dos resíduos são autocorrelacionados em alguns deles. Este comportamento, mostrado na Tabela 6 , indica a presença de heterocedasticidade condicional. 


\section{TABELA 6 - TESTE DE LJUNG-BOX PARA O QUADRADO DOS RESÍDUOS}

\begin{tabular}{lll}
\hline Parâmetros & \multicolumn{1}{c}{ A2 } & A4 \\
\hline$Q^{2}(1)$ & $(0,4064)$ & $(0,0039)^{\star \star \star}$ \\
$Q^{2}(2)$ & $(0,6986)$ & $(0,0048)^{\star \star \star}$ \\
$Q^{2}(3)$ & $(0,0028)^{\star \star \star}$ & $(0,0112)^{\star \star}$ \\
$Q^{2}(4)$ & $(0,0069)^{\star \star \star}$ & $(0,0229)^{\star \star}$ \\
\hline
\end{tabular}

Fonte: Dados da pesquisa. Entre parênteses, a probabilidade exata do teste. $\left({ }^{*}\right),\left(*^{*}\right)$ e $\left(*^{* *}\right)$ denotam a rejeição da hipótese nula de ausência de autocorrelação, respectivamente a 10\%, 5\% e 1\% de significância.

Em suma, observa-se que os resíduos apresentam heterocedasticidade condicional nas duas subamostras, A2 e A4, e de forma bem mais pronunciada neste último subperíodo. Logo, a caracterização fracionária na média não apresenta um bom ajuste. Deve-se, portanto, abandonar a estrutura de erros gaussianos, partindo para uma caracterização GARCH. Esta abordagem será considerada na seção seguinte.

\subsection{Resultados para o Modelo ARFIMA-FIGARCH}

A estimativa de um modelo ARFIMA-FIGARCH para a inflação brasileira, em particular para a subamostra A4, encontra-se sumariada na Tabela 7. Apesar de A2 também apresentar indício de integração fracionária, por ser uma subamostra com presença de inliers, como documentado em Cati et. al. (1999), não foi possível estimar um modelo ARFIMA-FIGARCH com o controle apropriado para essas características da série.

Por isso, a estimação de um modelo fracionário para a média e para a variância só foi possível na subamostra $\mathbf{A 4}$, com indícios de integração fracionária conforme se observa na Tabela 2 (sobrediferenciação), porém sem inliers ou mudanças estruturais.

Neste caso, o processo de seleção das ordens $p$ e $q$ do modelo baseou-se na minimização do critério de AIC e BIC (Critério de Informação Bayesiano) ${ }^{10}$ Considerouse uma distribuição t-student para os erros em vista da constatação de excesso de curtose para esse subperíodo $(=5,654)$. A estimativa para um modelo ARFIMAFIGARCH $\left(2, d_{m}, 2 ; 1, d_{n}, 1\right)$ encontra-se sumariada na Tabela 7.

10 Em vista da estrutura complexa do modelo, somente ordens de 1 a 3 foram avaliadas. O melhor ajuste foi obtido com a ordem $(2,2,1,1)$. As saídas do software estão disponíveis com os autores, sob demanda. 
TABELA 7 - MODELO ARFIMA-FIGARCH PARA A INFLAÇÃO BRASILEIRA

\begin{tabular}{|c|c|}
\hline Parâmetros & A4 \\
\hline$\hat{d}_{m}$ & $\begin{array}{c}0,2468^{\star \star \star} \\
(0,0000)\end{array}$ \\
\hline$\hat{\mu}$ & $0,2336^{* * *}$ \\
\hline$\hat{a}_{1}$ & $-0,3894^{* \star \star}$ \\
\hline$\hat{a}_{2}$ & $-0,0688^{* * *}$ \\
\hline$\hat{b}_{1}$ & $0,9013^{* * *}$ \\
\hline$\hat{b}$ & $0,3028^{\star \star \star}$ \\
\hline & $(0,0000)$ \\
\hline$\hat{d}_{v}$ & $0,4101^{\star \star \star}$ \\
\hline$\hat{\omega}$ & $1,7351^{\star \star \star}$ \\
\hline & $(0,0000)$ \\
\hline$\hat{\varphi}_{1}$ & $\begin{array}{l}0,0168^{\star \star \star} \\
(0,0000)\end{array}$ \\
\hline$\hat{\beta}_{1}$ & $\begin{array}{l}-0,2865^{\star \star *} \\
(0,0000)\end{array}$ \\
\hline $\log \ell$ & $-132,4716$ \\
\hline AIC & 1,7934 \\
\hline $\mathrm{BIC}$ & 2,0048 \\
\hline$Q(4)$ & $(0,3205)$ \\
\hline$Q(10)$ & $(0,3680)$ \\
\hline $\mathrm{Q}(20)$ & $(0,4944)$ \\
\hline$Q^{2}(4)$ & $(0,1531)$ \\
\hline$Q^{2}(10)$ & $(0,4723)$ \\
\hline$Q^{2}(20)$ & $(0,9048)$ \\
\hline
\end{tabular}

Fonte: Dados da pesquisa. Probabilidade exata do teste entre parênteses. $\mathrm{Q}($.$) e \mathrm{Q}^{2}($.$) denotam as defasagens$ utilizadas no teste Ljung-Box para os resíduos e para o quadrado dos resíduos da regressão respectivamente. ${ }^{* *}$ denota significância estatística, a $1 \%$.

O novo ajuste indica que não só os resíduos como também os seus quadrados são não-autocorrelacionados. Ou seja, "eliminou-se" a heterocedasticidade condicional.

Observando-se os resultados expostos na Tabela 7, pode-se dizer, com relação à década de 1990 (A4), no período pós-Plano Real, que a taxa de inflação pode ser descrita como um processo fracionário de longa memória, indicando alta persistência e um lento decaimento hiperbólico, porém com caráter estacionário (reversível) tanto na média quanto na variância. 
Esse traço de estacionariedade contrasta com os achados de Cati et al. (1999) e Baillie et al. (1996b), relativos à década de 1980 até o último plano de estabilização anterior ao Real (Collor II). Para o período pós-Real, os impulsos inflacionários ou deflacionários (choques) podem ser tratados como uma influência de natureza transitória, porém com lento decaimento hiperbólico, sobre a média e a volatilidade da inflação brasileira.

Posto que o coeficiente fracionário da variância condicional é significativo, a memória de longo prazo da inflação está presente não somente sobre a média do processo, mas também sobre a volatilidade. Como os componentes autorregressivos e de médias móveis de primeira e segunda ordem são estatisticamente significativos, a memória inflacionária de curto prazo também é captada pelo modelo. ${ }^{11}$

Diante desses resultados, a hipótese de um comportamento defensivo e persistente por parte dos agentes econômicos, por longos períodos de tempo, parece uma boa descrição da realidade. Neste caso, isolada dos impulsos inflacionários (deflacionários), a explicação para a tendência inflacionária está associada ao comportamento defensivo dos agentes econômicos, procurando recompor seu pico de renda real obtido no passado, como presume a hipótese da inflação inercial, independentemente das expectativas. ${ }^{12}$

Em estudo anterior, Baillie et al. (1996b) estimam um modelo ARFIMA-GARCH para a inflação brasileira no período 1947-1990, concluindo pela não-estacionariedade $\left(\hat{d}_{m}=0,5980\right)$ (irreversibilidade). Este estudo constitui um passo à frente nessa direção, posto que a estimação de um modelo ARFIMA-FIGARCH permite a caracterização fracionária não apenas na média, mas também na variância do processo. Os resultados obtidos para o período 1994:08 - 2008:01 revelam a influência e a efetividade do Plano Real e reforçam a interpretação da inflação brasileira como um processo de longa memória, de natureza tipicamente inercial, isto é, de alta persistência no curto e no longo prazo, tanto na média quanto na variância, porém, com caráter predominantemente estacionário (reversível).

Constata-se que o processo inflacionário deixou de apresentar o caráter não estacionário na média, característico da década de 1980 e períodos precedentes, preservando o traço de um processo reversível de lento decaimento hiperbólico, uma vez que o coeficiente fracionário da média é estatisticamente significativo $\left(\hat{d}_{m}=0,2468\right)$.

11 Definir inércia inflacionária como um parâmetro autorregressivo de $\mathrm{l}^{\mathrm{a}}$ ordem (memória curta), como fazem Laurini e Vieira (2005), não é incompatível com a estimação de um modelo ARFIMA-FIGARCH, visto que este modelo capta tanto a memória de curto prazo quanto a memória de longo prazo do processo, representada pelo coeficiente $d$ para a média e para a variância. Ver Sowell (1992) e Baillie $e t$ al. (1996a).

12 Essa era uma das premissas teóricas que nortearam a elaboração e a implantação do Plano Real. Ver Bacha (1998). 
Conforme os resultados acima, após a sua implementação do Plano Real, uma parte importante da memória inflacionária, proveniente da década de 1980, deixou de existir.

Diante desses resultados, pode-se dizer que, a despeito da remoção de grande parte da memória inflacionária com a implantação do Plano Real, um resíduo de inércia ainda persiste conjuntamente com uma volatilidade de caráter estacionário. Neste caso, seria importante ter clareza sobre a relação entre essas variáveis, posto que a volatilidade pode ser interpretada como um indicador da incerteza ainda presente na economia brasileira.

Para alcançar esse objetivo, empregou-se o teste de causalidade de Granger (GRANGER, 1969; GRANGER et. al., 1998). A Tabela 8 apresenta os resultados do teste de causalidade para a taxa de inflação e sua volatilidade. ${ }^{13}$

\section{TABELA 8 - TESTES DE CAUSALIDADE DE GRANGER}

\begin{tabular}{lc}
\hline Teste & $\mathrm{A} 4$ \\
\hline Inflação defasada não causa volatilidade & $3,5781^{\star \star}$ \\
Volatilidade defasada não causa inflação & $15,4992^{\star \star \star}$ \\
\hline
\end{tabular}

Fonte: Dados da pesquisa. $\left({ }^{*}\right),\left({ }^{* *}\right)$ e $\left({ }^{* *}\right)$ denotam a rejeição da hipótese nula de que a variável "a” nãocausa Granger a variável "b”, respectivamente a $10 \%, 5 \%$ e $1 \%$ de significância.

Dos resultados apresentados na Tabela 8 , constata-se que, após a implantação do Plano Real, tende a ocorrer uma recíproca influência entre a taxa de inflação e a volatilidade do processo inflacionário. Isto é, os valores antecedentes da taxa de inflação melhoram a previsão da volatilidade e vice-versa.

Se for possível postular uma certa simetria a partir desses resultados, visto que essa influência é positiva, pode-se esperar para os próximos períodos, com a permanência da taxa de inflação nos atuais patamares, que a volatilidade também venha a permanecer em níveis reduzidos. Deste modo, isso refletirá um ambiente de menor incerteza e maior credibilidade para os agentes econômicos, favorecendo as expectativas de longo prazo e a estabilidade macroeconômica ainda em consolidação.

13 Por questão de espaço, os resultados dos pré-testes (testes de raiz unitária ADF e PP) requeridos para a escolha apropriada do modelo (GRANGER, 1969; GRANGER et. al., 1998) foram omitidos. Conforme os resultados dos testes, ADF e PP, constatou-se que ambas as variáveis (taxa de inflação e volatilidade) na subamostra A4 são I (0), por isso foi suficiente estimar a equação (5.1) de Granger (1969, p. 431). Em caso contrário, seriam necessários testes adicionais de cointegração visando a seleção de um dos dois modelos de causalidade apresentados em Granger et. al. (1998, p. 5). O critério de seleção utilizado nas defasagens foi o AIC. 


\section{CONSIDERAÇOEES FINAIS}

O objetivo principal deste estudo foi investigar a presença de memória longa nos dois primeiros momentos da inflação brasileira, representada pela variação percentual mensal do IGP-DI. A partir da estimação de um modelo ARFIMA-FIGARCH, constatou-se que, no período pós - Plano Real, a taxa de inflação pode ser descrita como um processo fracionariamente integrado tanto na média quanto na variância. Além do processo inflacionário apresentar uma componente inercial de curto prazo (memória curta), foi possível também detectar uma componente inercial de longo prazo, caracterizada pela presença de memória longa nos dois primeiros momentos da taxa de inflação.

Apesar de o caráter de irreversibilidade do processo ter deixado de existir após o Plano Real, um resíduo de inércia inflacionária (memória) ainda persiste na economia brasileira. Com relação à mútua influência da inflação e da volatilidade, pode-se presumir que, mantida sob controle, uma baixa taxa de inflação deverá acarretar uma volatilidade menor no futuro, refletindo um ambiente de menor incerteza para os agentes econômicos, favorecendo as perspectivas de longo prazo e a consolidação da estabilização.

Diante desses resultados, é lícito observar que uma estratégia consistente no sentido de eliminar o resíduo inercial ainda presente na economia brasileira requer um trabalho conjunto e coordenado que transcende em alguma medida os domínios da autoridade monetária. Para tanto, torna-se necessário o redesenho do aparato institucional com a remoção das indexações formais (preços administrados por contrato) ainda presentes e preservadas pelo governo e também o ataque aos desequilíbrios reais, de caráter distributivo, que provocam o comportamento defensivo por parte dos agentes econômicos.

\section{REFERENCLIAS}

BACHA, E. L. O Plano Real: uma avaliação. In: MERCADANTE, A. (Org.). $O$ Brasil pós-Real: a política econômica em debate. Campinas: Unicamp-IE, 1998, p. 11-69.

BAILLIE, R. Long memory process and fractional integration in econometrics. Journal of Econometrics, 73, p. 5-59, 1996.

.; BOLLERSLEV, T.; MIKKELSEN, H. O. Fractionally Integrated Generalized Autoregressive Conditional Heteroskedasticity. Journal of Econometrics, 74, p. 3-30, 1996a. 
BAILlIE, R., CHUNG, C.-F; TIESLAU, M. A. Analyzing inflation by the fractionally integrated Arfima-Garch model. Journal of Applied Econometrics, 11, p. 23-40, $1996 b$.

BAILLIE, R.; HAN, Y.; KWON, T. Further long memory properties of inflationary shocks. Southern Economic Journal, v. 68, p. 496-510, 2002.

BANERJEE, A.; COCKERELL, L.; RUSSEL, B. An I(2) Analysis of inflation and the markup. Journal of Applied Econometrics, 16, p. 221-240, 2001.

BOGDANSKI, J.; TOMBINI, A. A.; WERLANG, S. R. C. Implementing Inflation Targeting in Brazil. Banco Central do Brasil, 2000. (Working Paper Series n. 1). Disponível em: <http://www.bcb.gov.br>. Acesso em: 09 ago. 2004.

BOLLERSLEV, T. Generalized autoregressive conditional heteroskedasticity. Journal of Econometrics, 31, 1986.

CAMPÊLO, A. K.; CRIBARI-NETO, F. Inflation inertia and 'inliers': the case of Brazil. Revista Brasileira de Economia, v. 57, n.4, p. 713-719, 2003.

CATI, R. C.; GARCIA, M. G. P.; PERRON, P. Unit roots in the presence of abrupt governmental interventions with an application to Brazilian data. Journal of Applied Econometrics, 14, p. 27-56, 1999.

CRIBARI-NETO, F.; CASSIANO, K. Uma análise da dinâmica inflacionária brasileira. Revista Brasileira de Economia, v.59, n. 4, p. 535-566, 2005.

FASOLO, A. M.; PORTUGAL, M. S. Imperfect rationality and inflationary inertia: a new estimation of the Phillips Curve for Brazil. UFRGS/PPGE, 2003. (Texto para Discussão). Disponível em: < http://www.ufrgs.br/ppge> . Acesso em: 10 out. 2004.

FIGUEIREDO; F. M. R.; FERREIRA, T. P. Os preços administrados e a inflação no Brasil. Brasília, 2002. (Trabalhos para Discussão n. 59). Disponível em: < http:// www.bcb.gov.br>. Acesso em: 10 mar. 2004.

FRIEDMAN, M. Inflation and Unemployment. Nobel Memorial Lecture, 1976. Disponível em: < http://nobelprize.org/nobel_prizes/economics/laureates/1976/ friedman-lecture.html>. Acesso em: 12 maio 2007.

GRANGER, C. W. Investigating causal relations by econometric models and by cross-spectral methods. Econometrica, v. 37, n. 3, p. 424-438, 1969.

. Long memory relationships and the aggregation of dynamic models. Journal of Econometrics, 14, p. 227-238, 1980.

. Some properties of time series data and their use in econometric model Specification. Journal of Econometrics, 16, p. 121-130, 1981.

; JOYEUX, R. An introduction to long memory time series models and fractional differencing. Journal of Time Series Analysis, 1, p. 5-39, 1980.

GRANGER, C. W. What we learning about the Long-Run?, Economic Journal, v. 103, n. 417, p. 307-317, 1993. 
; HUANG, B.-N.; YANG, C. W. A bivariate causality between stock prices and exchange rates: evidence from recent Asia flu. San Diego: University of California, 1998. (Discussion Paper, n. 98-09). Disponível em: < http://www. econ.ucsd.edu/papers/files/ucsd9809.pdf>. Acesso em: 21 jun. 2004.

HASLETT, J., RAFTERY A. Space-time modelling with long-memory dependence: assessing Ireland's wind power resource (with Discussion). Applied Statistics, 38, p. 1-50, 1989.

HOSKING, J. R. M. Fractional differencing. Biometrika, 68, p. 165-176, 1981.

KWIATKOWSKI, D.; PHILLIPS, P. C. B.; SCHMIDT, P.; SHIN, Y. Testing the null hypothesis of stationarity against the alternative of a unit root. Journal of Econometrics, 54, 1992.

LAURINI, M.; VIEIRA, H. A dynamic econometric model for inflationary inertia in Brazil. IBMEC, 2005. (No prelo).

LOPES, F. L. Inflação inercial, hiperinflação e desinflação: notas e conjecturas. Revista de Economia Política, v. 5, n. 2, p. 135-151, 1985.

MAIA, A.; CRIBARI NETO, F. Dinâmica inflacionária brasileira: resultados de auto-regressão quantílica. Revista Brasileira de Economia, v. 60, n. 2, p. 153-165, 2006.

MODIANO, E. A dinâmica de salários e preços na economia brasileira: 1966-1981. Pesquisa e Planejamento Econômico, v. 15, n. 1, p. 39-68, 1983.

MONTAÑÉS, A.; OLLIQUI, I.; CALVO, E. Selection of the break in the Perrontype tests. Journal of Econometrics, 129, p. 41-64, 2005.

PHILLIPS, P. C. B.; PERRON, P. Testing for a unit root in time series regression, Biometrika, v. 75, n. 2, 1988.

REISEN, V. A. Estimation of the fractional difference parameter in the ARFIMA $(\mathrm{p}, \mathrm{d}, \mathrm{q})$ model using the smoothed periodogram. Journal Time Series Analysis, v. 15, n.1, 1994.

RESENDE, A. L. A moeda indexada: uma proposta para eliminar a inflação inercial. Revista de Economia Politica, v. 5, n.2, p. 130-134, 1985.

SCHWERT, G. Tests for unit roots: a Monte Carlo investigation. Journal of Business and Economic Statistics, v. 7, 1989.

SIMS, C. A. Bayesian skepticism on unit root econometrics. Journal of Economic Dynamics and Control, v. 12, 1988.

SOWELL, F. The fractional unit root distribution. Econometrica, v. 58, n. 2, p. 495-505 1990.

. Modeling long-run behavior with the fractional ARIMA model. Journal of Monetary Economics, 2, p. 277-302, 1992.

TEJADA, C.; PORTUGAL, M. Credibilidade e inércia inflacionária no Brasil: 19861998. Estudos Econômicos, v. 31, n. 3, p. 459-494, jul.-set. 2001. 
ZIVOT, E.; ANDREWS, D. Further evidence on the great crash, the oil-price shock, and the unit-root hypothesis. Journal of Business \& Economic Statistics, v. 10, n. 3, p. 251-270, 1992. 